\title{
Low Complexity Sparse Channel Estimation for Wideband mmWave Systems: Multi-Stage Approach
}

\author{
Mojtaba Jahandideh*, Mohammad Moltafet*, Marian Codreanu ${ }^{\dagger}$, Matti Latva-aho* \\ ${ }^{*}$ Centre for Wireless Communications (CWC), University of Oulu, Finland \\ Email: firstname.lastname@oulu.fi \\ ${ }^{\dagger}$ Department of Science and Technology, Linköping University, Sweden \\ Email: marian.codreanu@liu.se
}

\begin{abstract}
We consider the problem of channel estimation in hybrid transceiver architectures operating in millimeter wave (mmWave) band. Due to the dynamic features of the environment and the sensitivity of mmWave bands to blockage and deafness, it is important to estimate mmWave channels with a low complexity and high performance algorithm. In this regard, we exploit the sparse structure of the frequency-selective mmWave channels and formulate the channel estimation problem as a sparse signal reconstruction in frequency domain. In order to solve the estimation problem, we propose a multi-stage based low complexity algorithm. Simulation results show that the proposed algorithm significantly reduces the computational complexity while preserving the quality of the estimation.
\end{abstract}

Index Terms- Millimeter waves, channel estimation, compressed sensing.

\section{INTRODUCTION}

Due to the explosive growth of high data rate services, scarcity of bandwidth is one the main challenges in the upcoming generation of wireless networks. Millimeter-wave (mmWave) band contains a huge unlicensed bandwidths which can be used to provide the high data rate services [1]. MmWave bands have directional feature which make them sparse in the spatial domain [2]. In addition, compressed sensing (CS) is a signal processing technique for efficiently acquiring and reconstructing a sparse signal. Therefore, CS is an appropriate tool to study the channel estimation problem in the mmWave based systems [2].

Multiple-input multiple-output (MIMO) architectures with large antenna arrays are practical in mmWave systems. Thanks to the small wavelength of mmWave, it is feasible to accommodate a large number of antenna elements in a physically limited space and reach gigabit-per-second data rates [1]. Due to the high path loss experienced in the mmWave frequencies, MIMO architectures with large array gain are needed to ensure that sufficiently high signal power can be received for successful

This research has been financially supported by Business Finland, Nokia, MediaTek, Bittium, Keysight, and Kyynel (High5 project), Academy of Finland (grant no. 307492), and Academy of Finland 6Genesis Flagship (grant no. 318927). Additionally, M. Moltafet was supported by the Infotech Oulu under the PROVING research project, and M. Codreanu would like to acknowledge the support of the European Union's Horizon 2020 research and innovation programme under the Marie Sklodowska-Curie Grant Agreement No. 793402 (COMPRESS NETS). signal detection. Hybrid MIMO structure turns out to be a more feasible solution because the cost and power consumption of a fully digital implementation is prohibitive at these frequencies. In the hybrid MIMO structure, antenna elements are grouped into analog subarrays and only a phase shifter is dedicated to a single antenna element and all the rest of the components are shared by all antenna elements in a subarray. Such a structure can largely reduce the cost and complexity with a significantly reduced number of hardware components [2]-[4].

Channel estimation, a critical task in any coherent communication system, is of a paramount importance in the mmWave band [5]. We need to acquire accurate mmWave channel state information (CSI) to fully exploit the potential advantages of hybrid mmWave systems, which is a challenging problem [6]. This problem was investigated in a significant number of papers; however, most of them, see, e.g., [5], [7]-[11], assumed a narrowband channel model and it is the main limitation of these works. However, since mmWave systems have wide bandwidth, developing efficient mmWave channel estimation for frequency-selective channels is essential. In [12], a distributed grid matching algorithm was proposed to estimate the frequency selective mmWave channels. Also, authors in [13], develop a sparse formulation and CS-based solution for the wideband mmWave channel estimation problem for hybrid architectures. However, the high computational complexity is the main drawback of these works.

In this paper, we develop a CS-based channel estimation for frequency-selective mmWave hybrid MIMO-OFDM systems exploiting the sparse nature of mmWave channels. Additionally, we formulate a sparse signal recovery problem in frequency domain for channel estimation. The main contributions of this paper is developing a multi-stage solution for the proposed optimization problem which considerably decreases the computational complexity while preserving the quality of recovery.

Notations: We use $\mathbf{A}^{*}, \overline{\mathbf{A}}$ and $\mathbf{A}^{T}$ to denote the conjugate transpose, conjugate and transpose of a matrix $\mathbf{A}$, respectively. The $(m, n)$-th entry of a matrix $\mathbf{A}$ is denoted using $[\mathbf{A}]_{m, n}$. Similarly, $[\mathbf{a}]_{m}$ denotes the $m$-th entry of a column vector $\mathbf{a}$. Also, $[\mathbf{A}]_{r,:}$ and $[\mathbf{A}]_{:, c}$ are the $r$ th row and $c$ th column of the matrix $\mathbf{A}$, respectively and $[\mathbf{A}]_{:, \mathcal{I}}$ is a sub-matrix of a matrix 


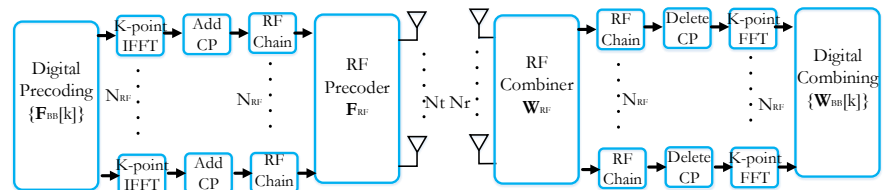

Fig. 1: Illustration of the OFDM based hybrid MIMO architecture with analog/digital precoding and combining.

A that only contains the columns whose elements are included in a set of column indices $\mathcal{I}$. The ceiling operator is denoted by $\lceil\cdot\rceil$ and $\bmod (a, b)$ is the remainder of $a$ when divided by $b$.

\section{SySTEM Model}

Consider the mmWave MIMO-OFDM system in Fig.1, where the the base station (BS) employs $N_{\mathrm{t}}$ antennas and $N_{\mathrm{RF}}$ RF chains with $N_{\mathrm{RF}} \leq N_{\mathrm{t}}$ to communicate with a user equipment (UE) with $N_{\mathrm{r}}$ antennas and $N_{\mathrm{RF}}$ RF chains with $N_{\mathrm{RF}} \leq N_{\mathrm{r}}$. The number of RF chains at the UE is usually less than that of the BS's, however, for simplicity of exposition we assume that they are equal. In order to realize the spatial multiplexing with low energy consumption and hardware cost, the hybrid analog-digital architecture is exploited at both the BS and the UE. At the BS, the training sequence $\mathbf{s}[k] \in \mathbb{C}^{N_{\mathrm{RF}} \times 1}$ is first precoded using a digital precoding matrix $\mathbf{F}_{\mathrm{BB}}[k] \in$ $\mathbb{C}^{N_{\mathrm{RF}} \times N_{\mathrm{RF}}}$ at each subcarrier $k=1, \ldots, K$. After that, the symbol blocks are transformed into the time domain using $N_{\mathrm{RF}}$ $K$-point IFFT's. Then, a cyclic prefix is added to the symbol blocks before applying RF precoding matrix $\mathbf{F}_{\mathrm{RF}} \in \mathbb{C}^{N_{\mathrm{t}} \times N_{\mathrm{RF}}}$. Note that the RF precoding matrix $\mathbf{F}_{\mathrm{RF}}$ is assumed to be frequency-flat, while the digital precoder is different for every subcarrier [14]. At the UE, applying RF combining matrix $\mathbf{W}_{\mathrm{RF}} \in \mathbb{C}^{N_{\mathrm{r}} \times N_{\mathrm{RF}}}$, the received signal is combined in the analog domain. After the cyclic prefix removal, the symbols are transformed to the frequency domain using $N_{\mathrm{RF}} K$-point FFT's. Then, digital combining matrix $\mathbf{W}_{\mathrm{BB}}[k] \in \mathbb{C}^{N_{\mathrm{RF}} \times N_{\mathrm{RF}}}$ is applied to the symbols at each subcarrier $k$. The received signal after processing at the $k$ th subcarrier can be expressed as [14] $\mathbf{y}[k]=\mathbf{W}_{\mathrm{BB}}^{*}[k] \mathbf{W}_{\mathrm{RF}}^{*} \mathbf{H}[k] \mathbf{F}_{\mathrm{RF}} \mathbf{F}_{\mathrm{BB}}[k] \mathbf{s}[k]+\mathbf{W}_{\mathrm{BB}}^{*}[k] \mathbf{W}_{\mathrm{RF}}^{*} \mathbf{n}[k]$,

where $\mathbf{H}[k] \in \mathbb{C}^{N_{\mathrm{r}} \times N_{\mathrm{t}}}$ denotes the channel matrix at subcarrier $k$, and $\mathbf{n}[k] \sim \mathcal{N}\left(\mathbf{0}, \sigma^{2} \mathbf{I}\right)$ is the circularly symmetric complex Gaussian distributed additive noise vector.

We consider geometric wideband mmWave channel model with $L$ paths to include the limited scattering characteristics of mmWave channels [2], [13], [15]. The $d$ th delay tap of the MIMO channel (for $d=0,1, \ldots, D$ ) can be written as [14]

$$
\mathbf{H}_{d}=\sum_{\ell=1}^{L} \alpha_{\ell} p_{\mathrm{rc}}\left(d T_{\mathrm{S}}-\tau_{\ell}\right) \mathbf{a}_{\mathbf{r}}\left(\phi_{\ell}\right) \mathbf{a}_{\mathrm{t}}^{*}\left(\theta_{\ell}\right)
$$

where $\alpha_{\ell} \in \mathbb{C}$ is the complex gain of the $\ell$ th channel path, $p_{\text {rc }}(\tau)$ denotes the band-limited pulse shaping filter response evaluated at time $\tau$, and $T_{\mathrm{S}}$ is the sampling time. Also, each cluster $\ell$ has a time delay $\tau_{\ell}$, and angles of arrival and departure (AoA/AoD), $\phi_{\ell}, \theta_{\ell} \in[0, \pi)$, and $\mathbf{a}_{\mathrm{r}}(\phi) \in \mathbb{C}^{N_{\mathrm{r}} \times 1}$ and $\mathbf{a}_{\mathrm{t}}(\theta) \in$ $\mathbb{C}^{N_{\mathrm{t}} \times 1}$ denote the antenna array response vectors of the UE and BS, respectively.

Given the geometric channel model in (2), the complex channel matrix at subcarrier $k$ can be written as [14], [13], [16]

$$
\begin{aligned}
\mathbf{H}[k] & =\sum_{d=0}^{D-1} \mathbf{H}_{d} e^{-j \frac{2 \pi k}{K} d} \\
& =\sum_{\ell=1}^{L} \alpha_{\ell} \mathbf{a}_{\mathbf{r}}\left(\phi_{\ell}\right) \mathbf{a}_{\mathrm{t}}^{*}\left(\theta_{\ell}\right) \sum_{d=0}^{D-1} p_{\mathrm{rc}}\left(d T_{\mathrm{S}}-\tau_{\ell}\right) e^{-j \frac{2 \pi k}{K} d},
\end{aligned}
$$

where $d$ is the delay tap index and $\mathbf{H}_{d}$ is given by (2). We can rewrite (3) as

$$
\mathbf{H}[k]=\sum_{\ell=1}^{L} \alpha_{\ell} \beta_{k, \ell} \mathbf{a}_{\mathbf{r}}\left(\phi_{\ell}\right) \mathbf{a}_{\mathbf{t}}^{*}\left(\theta_{\ell}\right),
$$

where $\beta_{k, \ell}=\sum_{d=0}^{D-1} p_{\mathrm{rc}}\left(d T_{\mathrm{S}}-\tau_{\ell}\right) e^{-j \frac{2 \pi k}{K} d}$. The channel model in (4) can be written in matrix form as

$$
\mathbf{H}[k]=\mathbf{A}_{\mathrm{r}} \mathbf{H}_{\mathrm{a}}[k] \mathbf{A}_{\mathrm{t}}^{*},
$$

where $\mathbf{H}_{\mathrm{a}}[k]=\operatorname{diag}\left(\alpha_{1} \beta_{k, 1}, \ldots, \alpha_{L} \beta_{k, L}\right)$ is a diagonal matrix, $\mathbf{A}_{\mathrm{r}}=\left[\mathbf{a}_{\mathrm{r}}\left(\phi_{1}\right), \ldots, \mathbf{a}_{\mathrm{r}}\left(\phi_{L}\right)\right] \in \mathbb{C}^{N_{\mathrm{r}} \times L}$, and $\mathbf{A}_{\mathrm{t}}=$ $\left[\mathbf{a}_{\mathbf{t}}\left(\theta_{1}\right), \ldots, \mathbf{a}_{\mathbf{t}}\left(\theta_{L}\right)\right] \in \mathbb{C}^{N_{\mathbf{t}} \times L}$. Further, we can rewrite (5) in vector form as

$$
\operatorname{vec}(\mathbf{H}[k])=\left(\overline{\mathbf{A}}_{\mathbf{t}} \otimes \mathbf{A}_{\mathbf{r}}\right) \operatorname{vec}\left(\mathbf{H}_{\mathrm{a}}[k]\right),
$$

where (6) follows from the identity, $\operatorname{vec}(\mathbf{A B C})=\left(\mathbf{C}^{T} \otimes\right.$ A) $\operatorname{vec}(\mathbf{B})$, and $\otimes$ denotes the Kronecker product.

\section{SPARSE Formulation IN THE FREQUENCY Domain}

By leveraging the sparse AoAs/AoDs of mmWave massive MIMO channels in the angular domain, we aim to formulate the compressed sensing problem in the frequency domain [6]. Accordingly, we define the matrices $\mathbf{A}_{\mathrm{rq}}=\left[\mathbf{a}_{\mathrm{r}}\left(\bar{\phi}_{1}\right), \ldots, \mathbf{a}_{\mathrm{r}}\left(\bar{\phi}_{G_{\mathrm{r}}}\right)\right] \in \mathbb{C}^{N_{\mathrm{r}} \times G_{\mathrm{r}}}$ and $\mathbf{A}_{\mathrm{tq}}=$ $\left[\mathbf{a}_{\mathbf{t}}\left(\bar{\theta}_{1}\right), \ldots, \mathbf{a}_{\mathbf{t}}\left(\bar{\theta}_{G_{\mathrm{t}}}\right)\right] \in \mathbb{C}^{N_{\mathrm{t}} \times G_{\mathrm{t}}}$, assuming that AoAs $\left(\bar{\phi}_{j}, j=\right.$ $\left.1, \ldots, G_{\mathrm{r}}\right)$ and AoDs $\left(\bar{\theta}_{k}, k=1, \ldots, G_{\mathrm{t}}\right)$ are drawn from uniform grids of size $G_{\mathrm{r}}$ and $G_{\mathrm{t}}$, respectively, with $G_{\mathrm{r}}, G_{\mathrm{t}} \geq L$. By ignoring the grid quantization error, the channel matrix model in (5) can be expressed using the extended virtual channel model defined in [2] as

$$
\mathbf{H}[k]=\mathbf{A}_{\mathrm{rq}} \mathbf{H}_{\mathrm{aq}}[k] \mathbf{A}_{\mathrm{tq}}^{*},
$$

where $\mathbf{H}_{\mathrm{aq}}[k] \in \mathbb{C}^{G_{\mathrm{r}} \times G_{\mathrm{t}}}$ is an $L$-sparse matrix, that is, most elements of $\mathbf{H}_{\mathrm{aq}}[k]$ are zero, and only $L$ elements associated with the AoAs and AoDs are non-zero. Note that unlike $\mathbf{H}_{\mathrm{a}}[k] \in \mathbb{C}^{L \times L}$ in (5) which is diagonal, $\mathbf{H}_{\mathrm{aq}}[k] \in \mathbb{C}^{G_{\mathrm{r}} \times G_{\mathrm{t}}}$ 
is not a diagonal matrix but a sparse matrix. By vectorizing $\mathbf{H}[k]$, we can further obtain

$$
\mathbf{h}[k]=\operatorname{vec}(\mathbf{H}[k])=\left(\overline{\mathbf{A}}_{\mathrm{tq}} \otimes \mathbf{A}_{\mathrm{rq}}\right) \mathbf{h}_{\mathrm{aq}}[k],
$$

where $\mathbf{h}_{\mathrm{aq}}[k]=\operatorname{vec}\left(\mathbf{H}_{\mathrm{aq}}[k]\right) \in \mathbb{C}^{G_{\mathrm{r}} G_{\mathrm{t}} \times 1}$ is an $L$-sparse vector.

Similar to the general expression in (1), we can write the received signal at the $k$ th subcarrier of the $m$ th OFDM symbol during the training phase as

$$
\begin{gathered}
\mathbf{y}_{m}[k]=\mathbf{W}_{\mathrm{BB}, m}^{*}[k] \mathbf{W}_{\mathrm{RF}, m}^{*} \mathbf{H}[k] \mathbf{F}_{\mathrm{RF}, m} \mathbf{F}_{\mathrm{BB}, m}[k] \mathbf{s}_{m}[k] \\
+\mathbf{W}_{\mathrm{BB}, m}^{*}[k] \mathbf{W}_{\mathrm{RF}, m}^{*} \mathbf{n}_{m}[k],
\end{gathered}
$$

We assume that the channel coherence time is large and the channel can be considered constant for several consecutive OFDM symbols. Using (8) in (9), we have

$$
\begin{aligned}
\mathbf{y}_{m}[k] & =\left(\mathbf{f}_{m}^{T}[k] \otimes \mathbf{W}_{m}^{*}[k]\right) \cdot \operatorname{vec}(\mathbf{H}[k])+\overline{\mathbf{n}}_{m}[k] \\
& =\left(\mathbf{f}_{m}^{T}[k] \otimes \mathbf{W}_{m}^{*}[k]\right)\left(\overline{\mathbf{A}}_{\mathrm{tq}} \otimes \mathbf{A}_{\mathrm{rq}}\right) \mathbf{h}_{\mathrm{aq}}[k]+\overline{\mathbf{n}}_{m}[k] \\
& \stackrel{(a)}{=}\left(\mathbf{f}_{m}^{T}[k] \overline{\mathbf{A}}_{\mathrm{tq}} \otimes \mathbf{W}_{m}^{*}[k] \mathbf{A}_{\mathrm{rq}}\right) \mathbf{h}_{\mathrm{aq}}[k]+\overline{\mathbf{n}}_{m}[k] \\
& =\mathbf{\Psi}_{m}[k] \mathbf{h}_{\mathrm{aq}}[k]+\overline{\mathbf{n}}_{m}[k],
\end{aligned}
$$

where

$$
\begin{aligned}
& \mathbf{f}_{m}[k]=\mathbf{F}_{\mathrm{RF}, m} \mathbf{F}_{\mathrm{BB}, m}[k] \mathbf{s}_{m}[k] \in \mathbb{C}^{N_{\mathrm{t}} \times 1}, \\
& \mathbf{W}_{m}[k]=\mathbf{W}_{\mathrm{RF}, m} \mathbf{W}_{\mathrm{BB}, m}[k] \in \mathbb{C}^{N_{\mathrm{r}} \times N_{\mathrm{RF}}}, \\
& \overline{\mathbf{n}}_{m}[k]=\mathbf{W}_{\mathrm{BB}, m}^{*}[k] \mathbf{W}_{\mathrm{RF}, m}^{*} \mathbf{n}_{m}[k] \in \mathbb{C}^{N_{\mathrm{RF}} \times 1}, \\
& \mathbf{\Psi}_{m}[k]=\left(\mathbf{f}_{m}^{T}[k] \overline{\mathbf{A}}_{\mathrm{tq}} \otimes \mathbf{W}_{m}^{*}[k] \mathbf{A}_{\mathrm{rq}}\right) \in \mathbb{C}^{N_{\mathrm{RF}} \times G_{\mathrm{r}} G_{\mathrm{t}}},
\end{aligned}
$$

and the equality $(a)$ holds because $(\mathbf{A} \otimes \mathbf{B})(\mathbf{C} \otimes \mathbf{D})=\mathbf{A C} \otimes$ BD [17].

Additionally, by stacking the received training signals of $M$ successive OFDM symbols, each with different precoders and combiners, we can write the following sparse formulation for the $k$ th subcarrier

$$
\tilde{\mathbf{y}}[k]=\tilde{\mathbf{\Psi}}[k] \mathbf{h}_{\mathrm{aq}}[k]+\tilde{\mathbf{n}}[k],
$$

where $\tilde{\mathbf{y}}[k]=\left[\mathbf{y}_{1}^{T}[k], \mathbf{y}_{2}^{T}[k], \cdots, \mathbf{y}_{M}^{T}[k]\right]^{T} \in \mathbb{C}^{M N_{\mathrm{RF}} \times 1}$ denotes the aggregate measurement signal, $\tilde{\boldsymbol{\Psi}}[k]=$ $\left[\boldsymbol{\Psi}_{1}^{T}[k], \mathbf{\Psi}_{2}^{T}[k], \cdots, \mathbf{\Psi}_{M}^{T}[k]\right]^{T} \in \mathbb{C}^{M N_{\mathrm{RF}} \times G_{\mathrm{r}} G_{\mathrm{t}}}$ is the aggregate sensing matrix, and $\tilde{\mathbf{n}}[k]=\left[\overline{\mathbf{n}}_{1}^{T}[k], \overline{\mathbf{n}}_{2}^{T}[k], \cdots, \overline{\mathbf{n}}_{M}^{T}[k]\right]^{T} \in$ $\mathbb{C}^{M N_{\mathrm{RF}} \times 1}$ denotes the aggregate additive white Gaussian noise (AWGN) signal.

\section{Multi-Stage Based Compressed Sensing Channel Estimation}

In conventional algorithms, in order to precisely estimate the mmWave channels from (12), the number of training OFDM symbols, $M$, should be large enough to provide sufficiently many independent equations and enable one to solve the estimation problem [5], [12]. Luckily, we can use the compressed sensing theory to estimate channels at each subcarrier with much reduced pilot overhead due to the sparsity of mmWave

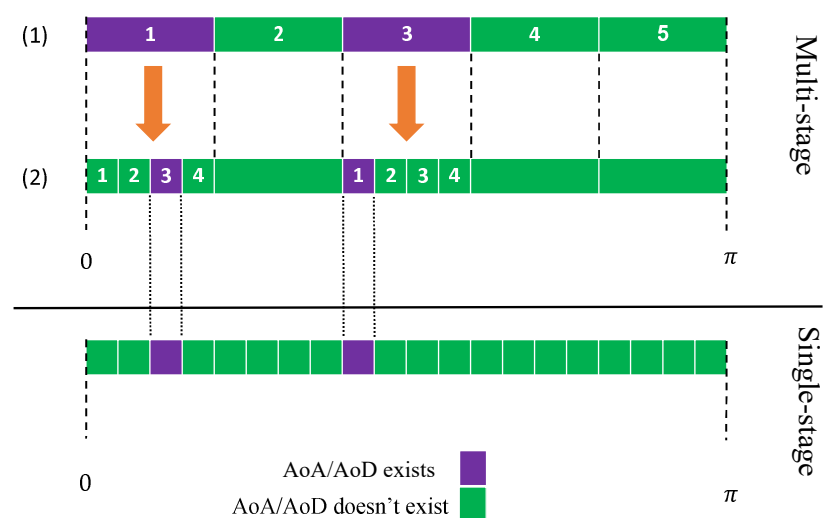

Fig. 2: The multi-stage and single-stage approaches. (Up): A multi-stage approach with two stages. Stage (1) is a coarse grid with 5 equal segments. At stage (2), the grid refines only around the regions that the AoAs/AoDs exist, in this example we divide them into 4 equal segments. (Down): Single-stage approach whit the same final resolution which searches through the whole segments.

channels. We can find the estimated channel vector $\hat{\mathbf{h}}_{\mathrm{aq}}[k]$ by solving the following sparse reconstruction problem

$$
\min \left\|\hat{\mathbf{h}}_{\mathrm{aq}}[k]\right\|_{0} \quad \text { subject to }\left\|\tilde{\mathbf{y}}[k]-\tilde{\mathbf{\Psi}}[k] \hat{\mathbf{h}}_{\mathrm{aq}}[k]\right\|_{2}^{2} \leq \delta,
$$

where $\|\cdot\|_{0}$ denotes the number of nonzero entries in a vector, and $\|\cdot\|_{2}$ is the $\ell_{2}$ norm. Also, $\delta$ is an adjustable threshold defining the maximum error between the measurement and the received signal assuming the reconstructed channel between the BS and the UE. From (13), the estimated channel for the $k$ th subcarrier is given by

$$
\hat{\mathbf{H}}[k]=\mathbf{A}_{\mathrm{rq}} \hat{\mathbf{H}}_{\mathrm{aq}}[k] \mathbf{A}_{\mathrm{tq}}^{*},
$$

where $\hat{\mathbf{H}}_{\mathrm{aq}}[k]=\operatorname{vec}^{-1}\left(\hat{\mathbf{h}}_{\mathrm{aq}}[k]\right)$.

There are a great variety of algorithms to find approximate solutions for (13). However, since the AoAs and AoDs are generated from the continuous uniform distribution, we need to increase the number of grid points $G_{\mathrm{r}}$ and $G_{\mathrm{t}}$ to improve the estimation performance which leads to heavy computational load. Therefore, we adopt a multi-stage approach to reduce the computational complexity of the reconstruction algorithm. To this end, we start with a coarse grid (or coarse resolution to estimate AoAs and AoDs) and only increase the resolution around the regions where the AoAs and AoDs are present. An example of this procedure is shown in Fig. 2.

\section{A. Case Study: MS-OMP}

The proposed multi-stage approach is a general approach and can be applied to different CS reconstruction algorithms. Here, we apply this approach to the well known Orthogonal Matching Pursuit (OMP) algorithm to solve (13). The multi-stage (MS)OMP algorithm is summarized in Algorithm 1, where, for 
simplicity of exposition, we assume that $G_{\mathrm{r}}=G_{\mathrm{t}}=G_{s}$, where $G_{s}$ denotes the size of grids at the stage $s$. At each iteration $t$, this algorithm adaptively refines the grid to achieve better precision. The initial sensing matrix for each subcarrier and the grid size are set to $\tilde{\mathbf{\Psi}}_{0}[k] \in \mathbb{C}^{M N_{\mathrm{RF}} \times G_{0}^{2}}$ and $G_{0}$, respectively. Also, the index of the stage is set to $s=1$. Then, the column of $\tilde{\boldsymbol{\Psi}}_{0}[k]$ which is most strongly correlated with the residual $\mathbf{r}_{t-1}[k]$ is chosen (step 8). In step 9, the indices of $\mathrm{AoD}$ and AoA corresponding to the selected column in previous step are obtained. Next, the grid size is set to $G_{s}$ and the matrices $\mathbf{A}_{\mathrm{tq}, s}$ and $\mathbf{A}_{\mathrm{rq}, s}$ are updated so that increase the angular resolution only around the regions where the AoDs and AoAs are present (steps 10-12). After forming the new sensing matrix $\tilde{\Psi}_{s}[k]$, the fine AoD and AoA pair index is obtained (steps 13-14). Then, we set $s=s+1$ and return to step 9 until the desired resolution is reached. The fine AoD/AoA pair set, $\mathcal{I}_{t}$, is updated in step 17 and in the next step, the channel gains are obtained by evaluating the least square solution of $\tilde{\mathbf{y}}[k]=[\tilde{\mathbf{\Psi}}[k]]_{: \mathcal{I}_{t}} \mathbf{h}[k]$. In step 19 , the residual is updated by subtracting the contributions of the chosen column vectors to $\tilde{\mathbf{y}}[k]$. This procedure is repeated until meet the criteria in step 5, i.e., $\left\|\mathbf{r}_{t-1}[k]-\mathbf{r}_{t-2}[k]\right\|_{2}^{2}$ falls below the predetermined threshold $\delta$. Next, the algorithm constructs the vector $\hat{\mathbf{h}}_{\mathrm{aq}}[k]$ so that $\left[\hat{\mathbf{h}}_{\mathrm{aq}}[k]\right]_{i}=\left[\mathbf{h}_{t-1}[k]\right]_{i}$ for $i \in \mathcal{I}_{t-1}$ and $\left[\hat{\mathbf{h}}_{\mathrm{aq}}[k]\right]_{i}=0$, otherwise (step 22). Finally, the desired estimation for subcarrier $k$ is given by $\hat{\mathbf{H}}_{\mathrm{aq}}[k]=\operatorname{vec}^{-1}\left(\hat{\mathbf{h}}_{\mathrm{aq}}[k]\right)$, and the corresponding channel estimation is obtained according to (14).

\section{B. Designing the Training Signals, Precoding and Combining Matrices}

As stated in [18], a sensing matrix can achieve the good performance for sparse signal recovery if its elements follow an independent identically distributed (i.i.d.) Gaussian distribution. This help us to design the OFDM training signals and the precoding/combing matrices, appropriately. As discussed above, we assume that for $1 \leq m \leq M$ and $1 \leq k \leq K$, we have

$$
\begin{aligned}
& {\left[\mathbf{W}_{\mathrm{RF}, m}\right]_{p_{1}, q_{1}}=e^{j \phi_{p_{1}, q_{1}, m}^{1}}, \quad 1 \leq p_{1} \leq N_{\mathrm{r}}, \quad 1 \leq q_{1} \leq N_{\mathrm{RF}},} \\
& {\left[\mathbf{W}_{\mathrm{BB}, m}[k]\right]_{p_{2}, q_{2}}=e^{j \phi_{p_{2}, q_{2}, m, k}^{2}}, 1 \leq p_{2} \leq N_{\mathrm{RF}}, 1 \leq q_{2} \leq N_{\mathrm{RF}},} \\
& {\left[\mathbf{F}_{\mathrm{RF}, m}\right]_{p_{3}, q_{3}}=e^{j \phi_{p_{3}, q_{3}, m}^{3}}, \quad 1 \leq p_{3} \leq N_{\mathrm{t}}, \quad 1 \leq q_{3} \leq N_{\mathrm{RF}},} \\
& {\left[\tilde{\mathbf{s}}_{m}[k]\right]_{p_{4}}=e^{j \phi_{p_{4}, q_{4}, m, k}^{4}}, \quad 1 \leq p_{4} \leq N_{\mathrm{RF}},}
\end{aligned}
$$

where $\phi_{p_{1}, q_{1}, m}^{1}, \quad \phi_{p_{2}, q_{2}, m, k}^{2}, \phi_{p_{3}, q_{3}, m}^{3}, \quad$ and $\phi_{p_{4}, q_{4}, m, k}^{4}$ follow the i.i.d. uniform distribution $\mathcal{U}[0,2 \pi)$. Also, $\tilde{\mathbf{s}}_{m}[k]=$ $\mathbf{F}_{\mathrm{BB}, m}[k] \mathbf{s}_{m}[k](1 \leq m \leq M, 1 \leq k \leq K)$. Note that as we assumed before, different subcarriers have the same RF precoding/combining. Also, RF precoding/combining matrices should meet the constant modulus property [12].

\section{Numerical Results}

In this section, in addition to complexity discussion of the proposed algorithm, we examine the performance of the $\overline{\text { Algorithm } 1 \text { MS-OMP based wideband mmWave channel }}$ estimator

1: Input: Measurement signals $\tilde{\mathbf{y}}[k]$ and sensing matrices $\tilde{\boldsymbol{\Psi}}[k]$ in (12) $\forall k$, the number of total stages $S$, and a threshold $\delta$

2: $\mathcal{I}_{0}=\emptyset ; \quad \triangleright$ Empty set

3: $\mathbf{r}_{-1}[k]=\mathbf{0}, \mathbf{r}_{0}[k]=\tilde{\mathbf{y}}[k] ; \quad \triangleright$ Set the residuals

4: $t=1$; $\triangleright$ Set the index of iteration

5: While $\left\|\mathbf{r}_{t-1}[k]-\mathbf{r}_{t-2}[k]\right\|_{2}^{2}>\delta$ do

6: $s=1$; $\triangleright$ Set the index of stage

7: $\quad$ While $s \leq S$

8: $\quad j=\arg \max _{n=1, G^{2}}\left|\left(\left[\tilde{\mathbf{\Psi}}_{s-1}[k]\right]:, n\right)^{*} \mathbf{r}_{t-1}[k]\right| ; \quad \triangleright$

Find AoA/AoD pair

9: $\quad g_{s-1}^{\mathrm{AoD}}=\left[\frac{j}{G_{s-1}}\right], g_{s-1}^{\mathrm{AoA}}=\bmod \left(j-1, G_{s-1}\right)+1 ; \triangleright$

Obtain the indices of AoD and AoA corresponding to $j$

10: $\quad$ Set $G_{s}$ at the stage $s$

11: $\quad \mathbf{A}_{\mathrm{tq}, s}=\left[\mathbf{a}_{\mathrm{t}}\left(\bar{\theta}_{1}\right), \ldots \mathbf{a}_{\mathrm{t}}\left(\bar{\theta}_{g_{s}^{\mathrm{AoD}}}\right), \ldots, \mathbf{a}_{\mathrm{t}}\left(\bar{\theta}_{G_{s}}\right)\right] \triangleright$ update the transformation matrix where $\bar{\theta}_{g_{s}^{\mathrm{AoD}}} \in\left[\bar{\theta}_{g_{s-1}^{\mathrm{AoD}}-1}, \bar{\theta}_{g_{s-1}^{\mathrm{AoD}}+1}\right]$ with $g_{s}^{\mathrm{AoD}}=1, \ldots, G_{s}$.

12: $\quad \mathbf{A}_{\mathrm{rq}, s}=\left[\mathbf{a}_{\mathrm{r}}\left(\bar{\phi}_{1}\right), \ldots, \mathbf{a}_{\mathrm{r}}\left(\bar{\phi}_{g_{s}^{\mathrm{AoA}}}\right), \ldots, \mathbf{a}_{\mathrm{r}}\left(\bar{\phi}_{G_{s}}\right)\right] \triangleright$ update the transformation matrix where $\bar{\phi}_{g_{s}^{\mathrm{AoA}}} \in$ $\left[\bar{\phi}_{g_{s-1}^{\mathrm{AoA}}-1}, \bar{\phi}_{g_{s-1}^{\mathrm{AoA}}+1}\right]$ with $g_{s}^{\mathrm{AoA}}=1, \ldots, G_{s}$.

13: $\quad$ Form the sensing matrix $\tilde{\boldsymbol{\Psi}}_{s}[k]$ using $\mathbf{A}_{\mathrm{rq}, s}$ and $\mathbf{A}_{\mathrm{tq}, s}$ 14: $\quad j=\arg \max _{n=1, \ldots, G_{s}^{2}}\left|\left(\left[\tilde{\boldsymbol{\Psi}}_{s}[k]\right]:, n\right)^{*} \mathbf{r}_{t-1}[k]\right| ; \quad \triangleright$ Finding

the fine AoA/AoD pair index

15: $\quad$ Set $s=s+1$ and return to step 9

16: End While

17: $\quad \mathcal{I}_{t}=\mathcal{I}_{t-1} \cup\{j\} ; \quad \triangleright$ Updating AoA/AoD pair set

18: $\quad \mathbf{h}_{t}[k]=\arg \min _{\mathbf{h}[k]}\left\|\tilde{\mathbf{y}}[k]-[\tilde{\mathbf{\Psi}}[k]]:, \mathcal{I}_{t} \mathbf{h}[k]\right\|_{2} \quad \triangleright$ Channel gains estimation

19: $\quad \mathbf{r}_{t}=\tilde{\mathbf{y}}[k]-[\tilde{\boldsymbol{\Psi}}[k]]:, \mathcal{I}_{t} \mathbf{h}_{t}[k] \quad \triangleright$ Update the residual

20: $\quad t=t+1$; $\quad \triangleright$ Update the index of iterations

21: End While

22: $\left[\hat{\mathbf{h}}_{\mathrm{aq}}[k]\right]_{i}=\left[\mathbf{h}_{t-1}[k]\right]_{i}$ for $i \in \mathcal{I}_{t-1}$ and $\left[\hat{\mathbf{h}}_{\mathrm{aq}}[k]\right]_{i}=0$ otherwise

23: Output: The estimated channel vector $\hat{\mathbf{h}}_{\mathrm{aq}}[k], \forall k$

proposed channel estimation respect to different parameters through computer simulations.

\section{A. Parameters}

We summarize the typical parameters for our systems as follows. Both the BS and the UE are assumed to use uniform linear arrays, hence, $\mathbf{a}_{\mathbf{t}}(\theta)$ is defined as

$$
\mathbf{a}_{\mathrm{t}}(\theta)=\frac{1}{\sqrt{N_{\mathrm{t}}}}\left[1, e^{j \frac{2 \pi}{\lambda} d_{\mathrm{s}} \sin (\theta)}, \ldots, e^{j\left(N_{\mathrm{t}}-1\right) \frac{2 \pi}{\lambda} d_{\mathrm{s}} \sin (\theta)}\right]^{T},
$$

where $\lambda$ is the wavelength of the signal, and $d_{\mathrm{s}}$ denotes the physical separation of antennas with $d_{\mathrm{s}}=\lambda / 2$. For $\mathbf{a}_{\mathrm{r}}(\phi)$, we have the similar expression. The number of antennas is set to $N_{\mathrm{t}}=32$ at the BS and $N_{\mathrm{r}}=16$ at the UE. Both the BS and the UE have $N_{\mathrm{RF}}=4 \mathrm{RF}$ chains. For the purpose of 
simulation, the number of OFDM subcarriers is set to $K=8$, and the threshold is set to $\delta=10^{-4}$. Additionally, following parameters are assumed in generating the channels. The number of channel paths is assumed to be $L=2$, with delay $\tau_{\ell}$ chosen uniformly from $\left[0,(D-1) T_{\mathrm{S}}\right]$, with $T_{\mathrm{S}}=\frac{1}{1760} \mathrm{~s}$, which is similar to 802.11ad wireless standard, and the AoAs/AoDs are assumed to be uniformly distributed in $[0, \pi)$. For the pulse shaping function, $p_{\mathrm{rc}}(t)$, raised-cosine filter with rolloff factor $\beta=0.8$ is adopted [14], and the number of delay taps is assumed to be $D=5$. We assume that the MS-OMP algorithm has two stages with $\left(G_{0}, G_{1}\right)=(30,5)$. The grid points are uniformly distributed over $[0, \pi)$, thus, the MS-OMP algorithm has angular resolutions of $3^{\circ}$. Also, for the purpose of comparison, we consider an OMP algorithm which has the same angular resolutions, we do this by considering single-stage OMP with a uniformly distributed grid points with $G=60$. For performance evaluation of channel estimation we use the Normalized Mean Squared Error (NMSE) metric defined as

$$
\mathrm{NMSE}=\frac{\sum_{k=0}^{K-1}\|\hat{\mathbf{H}}[k]-\mathbf{H}[k]\|_{F}^{2}}{\sum_{k=0}^{K-1}\|\mathbf{H}[k]\|_{F}^{2}},
$$

which will be averaged over multiple channel realizations.

\section{B. Complexity Discussion}

In this subsection, we evaluate the complexity of the proposed MS-OMP method. We compare the order of complexity of the MS-OMP algorithm with the conventional OMP algorithm with the same final resolution. The complexity of two algorithms for each subcarrier are summarized in Table I.

Fig. 3 depicts the complexity of the proposed MS-OMP and conventional methods. As it is observed, by increasing the number of stages, for the same resolution, the complexity reduces surprisingly. For example, by increasing the number of stages from 1 to 2 , the order of complexity decreases by approximately $75 \%$.

\section{Performance Discussion}

Fig. 4 compares the NMSE of the proposed MS-OMP algorithm and the conventional OMP. As we can see, the two algorithms show similar performance characteristics but the former requires much less computation than the latter. Also, as we can see, the gap between the obtained NMSE for $M=10$ and $M=15$ for a given algorithm, increases as increasing the SNR. Fig. 5 shows the average NMSE of MS-OMP and OMP algorithms versus the number of training frames $M$. The number of training frames $M$ is increased from 2 to 20. Comparisons are provided for $\mathrm{SNR}=5 \mathrm{~dB}$ and 15 dB. As we can see from the figure, both of the algorithms have almost similar performance but the MS-OMP algorithm is much less computationally complex. Also, more number of training frames lead to better estimation error performance at the expense of higher signaling overhead. In Fig. 6, we see employing multiple RF chains at the BS and the UE can give

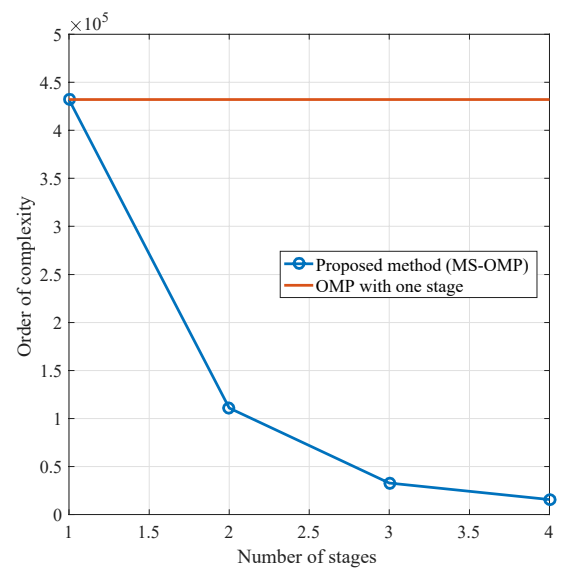

Fig. 3: Order of complexity as a function of the number of stages with $L=2, M=15, N_{\mathrm{RF}}=4, G=60$ and resolution 3 degree.

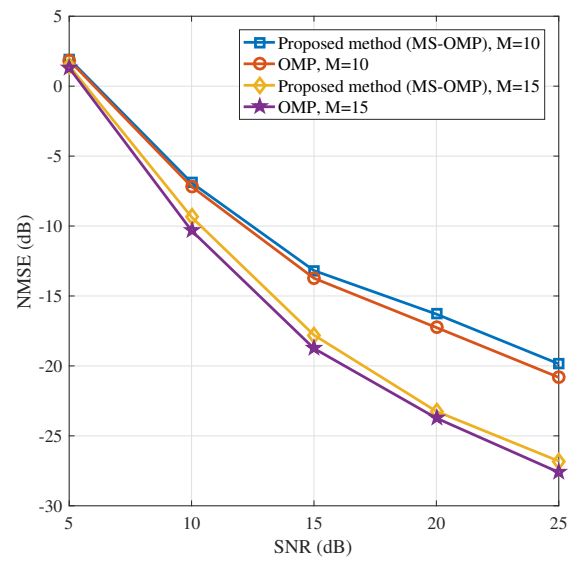

Fig. 4: Average NMSE as a function of (a) SNR for different training length $M$.

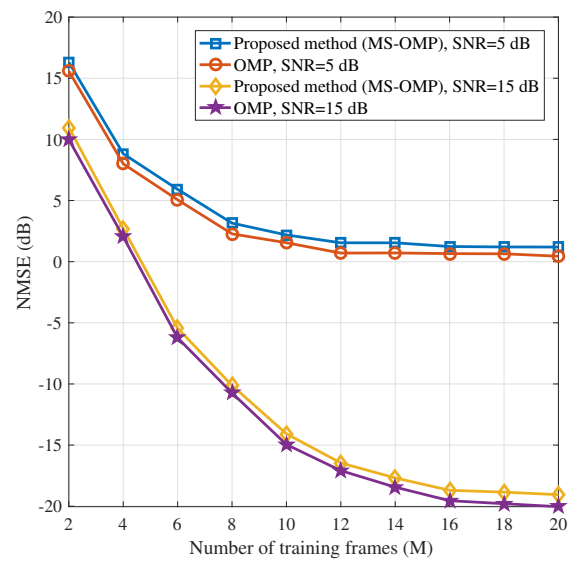

Fig. 5: Average NMSE as a function of the number of training frames $M$. 
TABLE I: Order of complexity with respect to different parameters

\begin{tabular}{|c|c|c|c|c|c|c|c|}
\hline Parameters & $L$ & $M$ & $N_{R F}$ & $G_{0}$ & $G_{1}$ & $G$ & Total order of complexity \\
\hline MS-OMP & 1 & 1 & 1 & 2 & 2 & - & $\mathcal{O}\left(L M N_{\mathrm{RF}}\left(G_{0}^{2}+G_{1}^{2}\right)\right)$ \\
\hline OMP & 1 & 1 & 1 & - & - & 2 & $\mathcal{O}\left(L M N_{\mathrm{RF}} G^{2}\right)$ \\
\hline
\end{tabular}

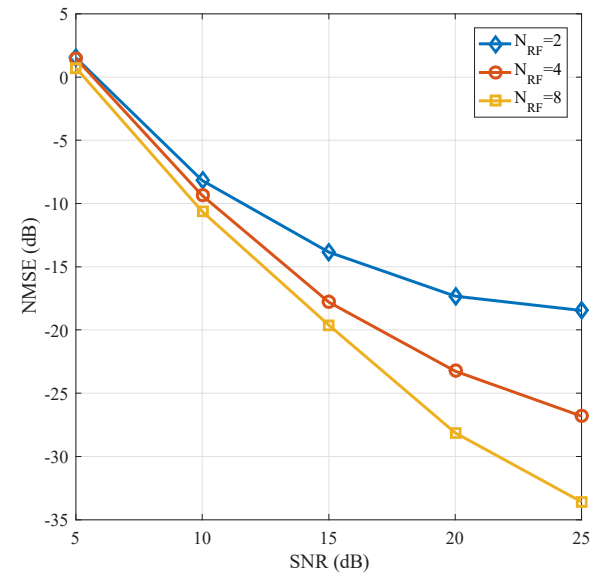

Fig. 6: Average NMSE as a function of SNR for different numbers of RF chains used at both the BS and the UE with $M=15$ and using MS-OMP algorithm.

good improvement in the estimation performance. In this figure we assume that $M=15$ frames are transmitted for training. The improvement in NMSE performance occurs thanks to a larger number of measurements per training frame which results in smaller estimation error via compressed sensing. So, by employing larger $N_{\mathrm{RF}}$ at the BS and the UE, the NMSE performance is improved and it is preferred to decrease the estimation error and to fully leverage the hybrid architecture in wideband mmWave systems.

\section{CONCLUSION}

In this paper, we proposed a sparse channel estimation algorithm for wideband mmWave systems with a hybrid architecture. Exploiting the sparsity of mmWave channels in angular domain, we formulated a compressed sensing problem that estimates the AoA, AoD, and the corresponding channel gain of each path in the frequency domain. In order to reduce the computational complexity of the spare recovery algorithm, we employed multi-stage approach. This algorithm starts with a coarse step and increases the resolution around the regions where the AoAs and AoDs are present. We applied our proposed method to the OMP reconstruction algorithm. Simulation results showed that the proposed MS-OMP algorithm has a very low complexity compare to the conventional OMP with the same resolution, while its performance is sufficiently closed to the conventional one.

\section{REFERENCES}

[1] T. S. Rappaport, R. W. Heath Jr, R. C. Daniels, and J. N. Murdock, Millimeter wave wireless communications. Pearson Education, 2014.

[2] R. W. Heath, N. Gonzalez-Prelcic, S. Rangan, W. Roh, and A. M. Sayeed, "An overview of signal processing techniques for millimeter wave MIMO systems," IEEE J. Sel. Top. Signal Process., vol. 10, no. 3, pp. 436-453, April 2016.

[3] X. Huang, Y. J. Guo, and J. D. Bunton, "A hybrid adaptive antenna array," IEEE Trans. Wirel. Commun., vol. 9, no. 5, pp. 1770-1779, May 2010.

[4] J. A. Zhang, X. Huang, V. Dyadyuk, and Y. J. Guo, "Massive hybrid antenna array for millimeter-wave cellular communications," IEEE Wirel. Commun., vol. 22, no. 1, pp. 79-87, March 2015.

[5] A. Alkhateeb, O. El Ayach, G. Leus, and R. W. Heath, Jr., "Channel estimation and hybrid precoding for millimeter wave cellular systems," IEEE J. Sel. Topics Signal Process., vol. 8, no. 5, pp. 831-846, October 2014.

[6] A. Alkhateeb, M. Jianhua, N. González-Prelcic, and R. W. Heath Jr., "MIMO precoding and combining solutions for millimeter-wave systems," IEEE Commun. Mag., vol. 52, no. 12, pp. 122-131, December 2014.

[7] J. Lee, G. Gye-Tae, and Y. H. Lee, "Exploiting spatial sparsity for estimating channels of hybrid MIMO systems in millimeter wave communications," in Proc. IEEE Globecom Communications, Austin, TX, December 2014, pp. 3326-3331.

[8] R. Méndez-Rial, C. Rusu, N. González-Prelcic, A. Alkhateeb, and R. W. Heath, "Hybrid MIMO architectures for millimeter wave communications: Phase shifters or switches?," IEEE Access, vol. 4, pp. 247-267, January 2016.

[9] A. Alkhateeb, G. Leus, and R. W. Heath Jr., "Compressed-sensing based multi-user millimeter wave systems: How many measurements are needed?", in Proc. IEEE Int. Conf. Acoust. Speech Signal Process.(ICASSP), Brisbane, April 2015, pp. 2909-2913.

[10] Y. Han and J. Lee, "Two-stage compressed sensing for millimeter wave channel estimation," in Proc, International Symposium Information Theory (ISIT), Barcelona, August 2016, pp. 860-864.

[11] H. Ghauch, T. Kim, M. Bengtsson, and M. Skoglund, "Subspace estimation and decomposition for large millimeter-wave MIMO systems," IEEE J. Sel. Top. Signal Process., vol. 10, no. 3, pp. 528-542, April 2016.

[12] Z. Gao, C. Hu, L. Dai and Z. Wang, "Channel estimation for millimeterwave massive MIMO with hybrid precoding over frequency-selective fading channels," IEEE Communications Letters, vol. 20, no. 6, pp. 12591262, June 2016.

[13] K. Venugopal, A. Alkhateeb, N. G. Prelcic, and R. W. Heath, Jr., "Channel estimation for hybrid architecture-based wideband millimeter wave systems," IEEE J. Sel. Areas Commun., vol. 35, no. 9, pp. 19962009, September 2017.

[14] A. Alkhateeb and R. W. Heath, Jr., "Frequency selective hybrid precoding for limited feedback millimeter wave systems," IEEE Trans. Commun., vol. 64, no. 5, pp. 1801-1818, May 2016.

[15] T. Rappaport, S. Sun, R. Mayzus, H. Zhao, Y. Azar, K. Wang, G. Wong, J. Schulz, M. Samimi, and F. Gutierrez, "Millimeter wave mobile communications for 5G cellular: It will work!" IEEE Access, vol. 1, pp. 335-349, May 2013.

[16] W. Bajwa, J. Haupt, A. Sayeed, and R. Nowak, "Compressed channel sensing: A new approach to estimating sparse multipath channels," Proceedings of the IEEE, vol. 98, no. 6, pp. 1058-1076, June 2010.

[17] K. M. Abadir and J. R. Magnus, Matrix algebra. Cambridge, U.K.: Cambridge Univ. Press, 2005.

[18] Y. C. Eldar and G. Kutyniok, Eds., Compressed Sensing: Theory and Applications. Cambridge, U.K.: Cambridge Univ. Press, May 2012. 